\title{
ENANTIOSELECTIVE EXTRACTION OF RACEMIC OFLOXACIN BY DI(2-ETHYLHEXYL)PHOSPHORIC ACID AND TARTARIC ACID DERIVATIVES AS MIXED COMPLEX CHIRAL SELECTORS
}

\author{
LINGXING TIAN, WEIJIE YANG, XIAOQING CHEN*, YIXI XIE \\ School of Chemistry and Chemical Engineering, Central South University, Changsha 410083, China \\ *Corresponding author: Prof. Xiaoqing Chen
}

(Received: November 23, 2009 - Accepted: April 15, 2011)

\begin{abstract}
The distribution behavior of ofloaxcin (OFLX) enantiomers in a two-phase system was examined using a new mixed complex chiral selector consisting of di(2-ethylhexyl) phosphoric acid (D2EHPA) and two tartaric acid derivatives with n-octanol as diluents. The influence of composition of mixed complex chiral selector, initial concentration of OFLX, pH of aqueous phase and temperature was investigated. A maximum enantioselectivity of 2.43 was obtained when the molar concentration ratio of L-(-)-DBTA to L-(-)-DTTA is $2: 3 \mathrm{~L}-(-)-D T T A$ concentration $0.18 \mathrm{~mol} / \mathrm{L}$, L-(-)-DBTA concentration $0.12 \mathrm{~mol} / \mathrm{L}$ ) and the pH of aqueous phase is 6.86 at $25^{\circ} \mathrm{C}$, with $D_{D}$ and $D_{L}$ up to a relative high value of 10.2 and 4.20 , respectively. The results indicated a useful way of searching new efficient chiral selectors and it was proved also helpful to optimize the extraction systems and realize the large-scale production of the pure isomer of racemic mixtures with extraction methods.
\end{abstract}

Key words: Enantioselective extraction, ofloxacin enantiomers, D2EHPA, tartaric acid derivative, complex chiral selectors.

\section{INTRODUCTION}

With the in-depth study of chiral drugs and the difference in their effects, the clinical significance of chiral drugs has been conceived of more and more. Enantiomers of chiral drugs have significant differences in the body of pharmacological activity, metabolism and toxicity ${ }^{1,2}$. The ever-increasing need of pure isomer of racemic drugs stimulates the development of chiral resolution technology, among of which, chromatography technique ${ }^{3,4}$ such as GC and LC, are the typical analytical methods of enantiomers. As a potential large scale production technique, solvent extraction has attracted a lot of researchers to make great efforts ${ }^{5,6}$. As is well known, the type of chiral selectors ${ }^{7-14}$ and their enantioselectivety play the most important role in the separation efficiency for enantioselective extraction. In relation to the conclusion reported by the previous work ${ }^{7}$, there formed a new complex chiral extractant when mixed O,O'-dibenzoyl-(2R,3R)-tartaric acid (L-(-)-DBTA) and di(2ethylhexyl)phosphoric acid (D2EHPA), and then both the distribution ratio and enantioselectivity are greatly improved by the formation of the complex instead of L-(-)-DBTA individually. But the enantioselectivity of this complex selectors can be somewhat low, and the large number of theoretical stages still needs to be further increased in these processes. In our previous work ${ }^{15}$, ${ }^{16}$, the resolution by mixed complex chiral selectors was investigated. Namely, tartaric acid derivatives (O,O'-dibenzoyl-(2R,3R)-tartaric acid, L-(-)-DBTA; O,O'-dibenzoyl-(2R,3R)-4-toluoyl tartaric acid, L-(-)-DTTA) were used with the most promising carriers for liquid membrane formulation di(2-ethylhexyl) phosphoric acid (D2EHPA), which has no chiral discrimination. It is found that both the distribution ratio and enantioselectivity may be increased compared with the individual complex chiral selectors. However, the chiral separation mechanisms of the extraction process are not clear and whether the method is effective to other racemates should be further investigated.

Therefore, based on the previous results, in the present work, enantioselective extraction of racemic ofloxacin (OFLX (Figure 1)) by D2EHPA and tartaric acid derivatives L-DBTA, L-(-)-DTTA as mixed complex chiral selectors was carried out. The effects of concentration proportion of L-()-DTTA and L-(-)-DBTA, initial concentration of OFLX, pH in the aqueous and temperature have been investigated. Additionally, the thermodynamic characteristics of the extraction process were analysised.

OFLX is chemically known as ( \pm )-9-fluoro-2,3-dihydro-3-methyl10-(4-methyl-1-piperazinyl)-7-oxo-7 H-pyrido[1,2,3-de]-1,4-benzoxazine6-carboxylic acid. It is obviously seen that the $D(L)$-OFLX contains one carboxylic group, and the amount of ionic OFLX would increase if the concentration of hydroxide $\left(\mathrm{OH}^{-}\right)$increased.<smiles>C[C@H]1COc2c(N3CCN(C)CC3)c(F)cc3c(=O)c(C(=O)O)cn1c23</smiles>

Figure 1: Molecular structure of rac-OFLX.

\section{EXPERIMENTAL}

\subsection{Material}

Racemic OFLX (purity $>99.0 \%$ ) and L-phenylalanine $[\alpha]_{D}^{20}=-34 \pm 1^{\circ}$ were purchased from Guangfu Institute of Fine Chemicals (Tianjin, China). $\mathrm{CuSO}_{4} \cdot 5 \mathrm{H}_{2} \mathrm{O}$ (purity $>99.0 \%$ ) was bought from Kemiou Chemical Reagents Co. Ltd (Tianjin,China). D2EHPA (CP) was supplied by Chemical Reagent Sinopharm Group Co. Ltd (Shanghai, China). $L$-(-)-DBTA and $L$-(-)-DTTA with purities $>99 \%$ were obtained from Lingxing \& Co. Inc. (Zhejiang, China). n-octanol (AP) was bought from Lingfeng Chemical Reagent Co. Ltd. (Shanghai, China). All other chemicals were of analytical-reagent grade.

\subsection{Apparatus}

The quantification of OFLX enantiomers in the aqueous phase was performed by HPLC using a UV detector (Shimadzu, Japan) at the UV wavelength of $300 \mathrm{~nm}$. The column was inertsil ODS-SP, $5 \mu \mathrm{m}$ particle size of the Packing Material, $150 \mathrm{~mm} \times 4.6 \mathrm{~mm}$ I.D. (Shimadzu, Japan).

2.3. Analysis

The mobile phase for OFLX enantiomers was 3mmol.L CuSO and $6 \mathrm{mmol} / \mathrm{L} L$-phenylalanine aqueous solution: methanol $(85: 15, \mathrm{v} / \mathrm{v})$ at a flow of $0.8 \mathrm{~mL} / \mathrm{min}$. The retention time of the $L$-enantiomer is less than that of the $D$-enantiomer (Figure 2). 


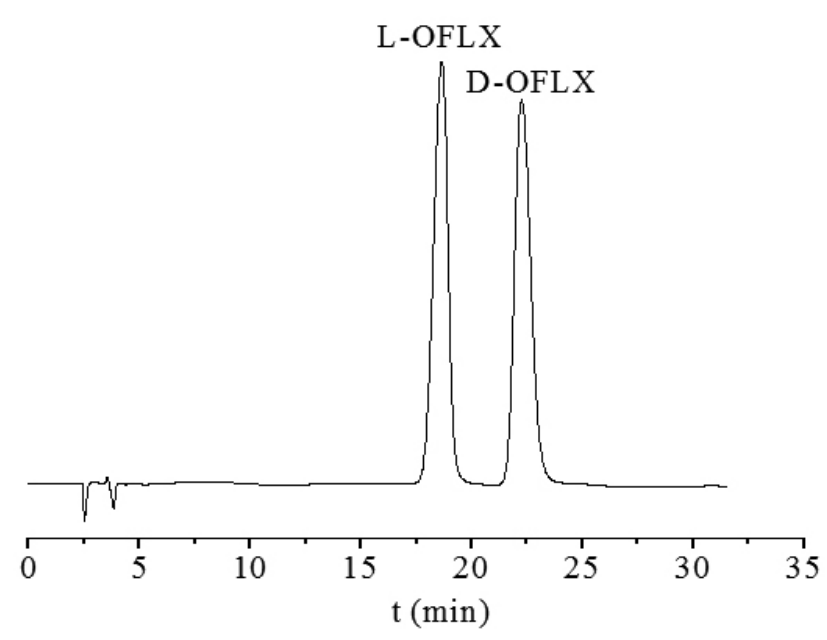

Figure 2: Chromatogram of $D, L-\mathrm{OFLX}$ in the aqueous phase.

\subsection{Procedure}

The aqueous phase was prepared by dissolving the racemic OFLX in water. The $\mathrm{pH}$ values were adjusted with a phosphate salt buffer solution and measured using a $\mathrm{pH}$ meter before extraction for each experiment. A tartaric acid derivative of complex chiral selector by tartaric acid derivative L-(-)DBTA $0.12 \mathrm{~mol} / \mathrm{L}, \mathrm{L}-(-)-\mathrm{DTTA} 0.18 \mathrm{~mol} / \mathrm{L})$ with D2EHPA $(0.3 \mathrm{~mol} / \mathrm{L})$ or mixed complex chiral selectors by tartaric acid derivatives with D2EHPA were used as the extractants and n-octanol as the diluent. Equal volumes (each $10 \mathrm{~mL}$ ) of the organic and aqueous phases were placed in a $50 \mathrm{~mL}$ glassstoppered flask together and shaken $30 \mathrm{~min}$ before placement into a water bath for phase separation at a fixed temperature $\left(25^{\circ} \mathrm{C}\right)$. After phase separation, the concentrations of OFLX enantiomers in the aqueous pase were analyzed by HPLC. Each experiment was tripled under identical conditions at least, and the standard deviation is in the range of $\pm 2 \%$. Since the change in volume is very small, it can be seen as negligible. The concentration of OFLX enantiomers in the organic phase is calculated by the subtractive method. The distribution ratio $(D)$ and enantioselectivity $(\beta)$ at equilibrium as the evaluated parameters are calculated from the equations $\mathrm{D}_{D(L)}=\mathrm{C}_{D(L)} \mathrm{o} / \mathrm{C}_{D(L)}$ a and $\beta=D_{D} / D_{L}$ respectively ${ }^{14,15}$, where $\mathrm{C}_{D(L)}$ is the mass concentration $(\mathrm{mmol} / \mathrm{L})$ of $D(L)$-OFLX in aqueous phase, $D_{D}(L)$ is the distribution ratio of $D$-enantiomer and $D_{L}$ is the distribution ratio of $L$-enantiomer.

\section{RESULTS AND DISCUSSION}

\subsection{The possible enantioseparation mechanism}

In this paper, the resolution of OFLX enantiomers by mixed complex chiral selectors was investigated. Namely, tartaric acid derivatives were used with D2EHPA, which can form mixed complex chiral selectors. The possible enantioseparation mechanism by mixed complex chiral selectors is similar to our previous work ${ }^{15}$. So far, few papers about the use of mixed complex resolution reagents in the field of chiral extraction were reported, thus the study of it will must enrich the theory of combinatorial resolution.

3.2. Effect of concentration proportion of L-(-)-DTTA and L-(-)-DBTA

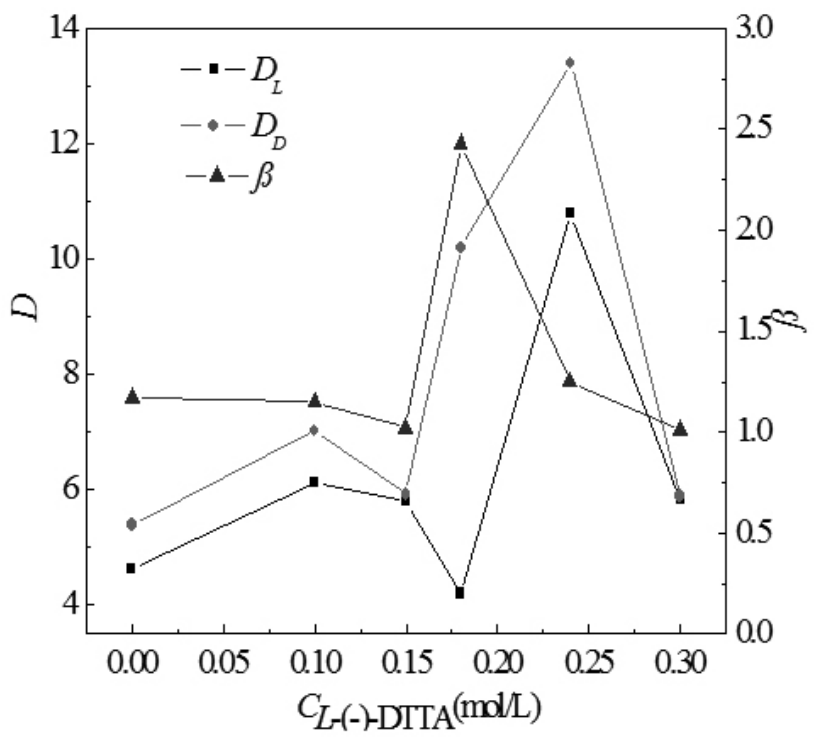

Figure 3: Influence of the initial concentration of L-(-)-DTTA on the distribution, enantioselectivity. Concentration of $D, L-O F L X, 200 \mathrm{ppm}$; D2EHPA, $0.3 \mathrm{~mol} / \mathrm{L}$, Total concentration of L-(-)-DTTA and L-(-)-DBTA, 0.3 $\mathrm{mol} / \mathrm{L}$; pHep, 6.86; and temperature, $25^{\circ} \mathrm{C}$.

As shown in Figure 3, with the increase of concentration of L-(-)-DTTA in the mixed extractant until $0.15 \mathrm{~mol} / \mathrm{L}$, that is, half of the total concentration, the distribution ratio of $D$-enantiomer and $L$-enantiomer are first increased and then decreased, and the similar variations take on with increasing concentration from 0.15 to $0.30 \mathrm{~mol} / \mathrm{L}$, while the enantioselectivity $\beta$ changes little on the concentration alterations and keeps a relative low value, except that a maximum enantioselectivity of 2.43 is obtained when the molar concentration ratio of L-(-)-DBTA to L-(-)-DBTA is 2:3 L-(-)-DBTA concentration $0.18 \mathrm{~mol} / \mathrm{L}$, L-(-)-DBTA concentration $0.12 \mathrm{~mol} / \mathrm{L}$ ) with $D_{D}$ and $D_{L}$ respectively up to a relative high value of 10.2 and 4.20 , which indicates that the enantioselectivity is apparently improved by the formation of the mixed complex chiral selectors instead of using D2EHPA and one of the tartaric acid derivatives.

3.3. Effect of initial concentration of ofloxacin

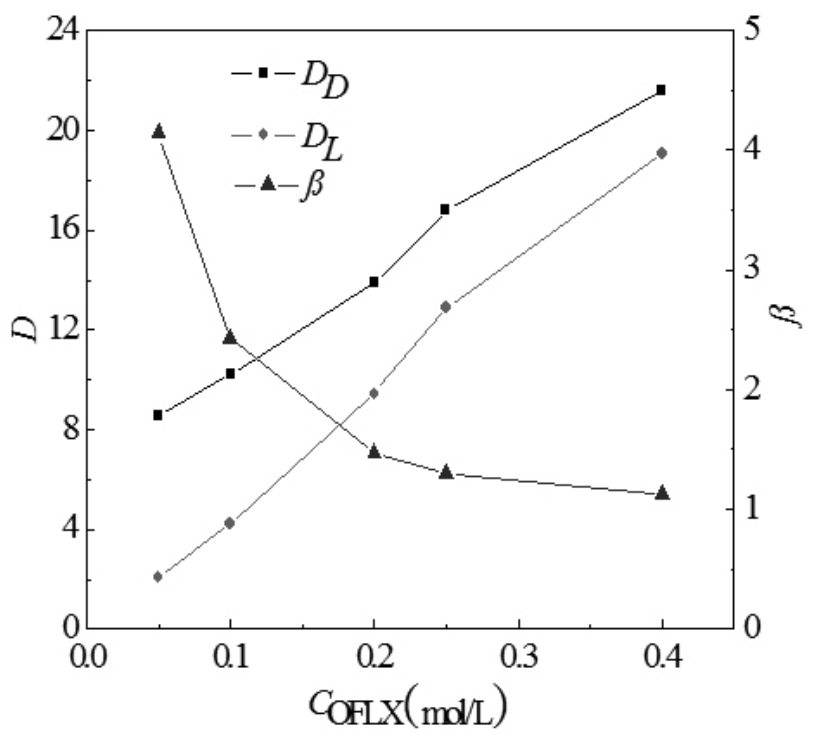

Figure 4: Influence of the initial concentration of $D, L$-OFLX on the distribution, enantioselectivity. D2EHPA, $0.3 \mathrm{~mol} / \mathrm{L}$; L-(-)-DTTA, $0.18 \mathrm{~mol} / \mathrm{L}$, L-(-)-DBTA, $0.12 \mathrm{~mol} / \mathrm{L}$; pHep, 6.86; temperature, $25^{\circ} \mathrm{C}$. 
Figure 4 shows the influence of the initial concentration of ofloxacin enantiomers on the distribution ratio of ofloxacin enatiomers and enantioselectivity. The distribution ratios of $D$-OFLX and $L$-OFLX are both enhanced with the increase of the initial concentration of ofloxacin. However, the value of enantioselectivity is relative higher at low concentrations, which indicates that a better enantioseparation efficiency can be obtained at low initial concentration.

\subsection{Effect of $\mathrm{pH}$}

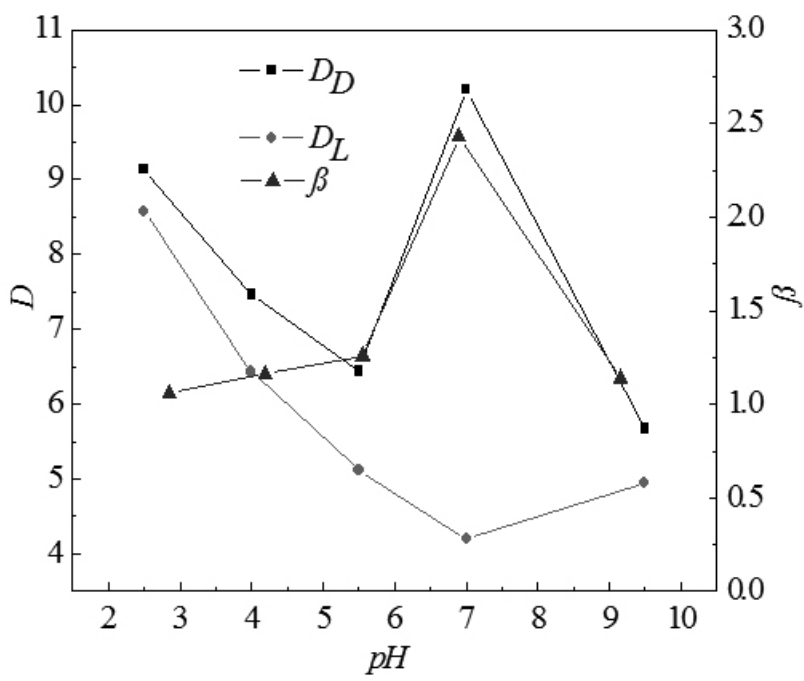

Figure 5: Influence of the $\mathrm{pH}$ on the distribution, enantioselectivity. Concentration of $D, L$-OFLX, 200ppm; D2EHPA, $0.3 \mathrm{~mol} / \mathrm{L}$; L-(-)-DTTA, $0.18 \mathrm{~mol} / \mathrm{L}, \mathrm{L}-(-)-\mathrm{DBTA}, 0.12 \mathrm{~mol} / \mathrm{L}$; temperature, $25^{\circ} \mathrm{C}$.

The effect of $\mathrm{pH}$ of the aqueous phase is shown in Figure 5. The distribution ratio of $L$-OFLX is first decreased with the increase of $\mathrm{pH}$ until 7.00 and then increased, while the distribution ratio of $D$-OFLX is first decreased and then increased to a maximum at $\mathrm{pH}=7.00$ and finally decreased. So, the enantioselectivity is first increased until it reaches to the maximum with the $\mathrm{pH}$ around 7.00, and then gradually descends. It is also able to see that no matter

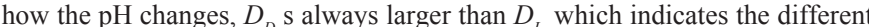
extractive capacity of the mixed extractants for the two types of enantiomers.

3.5. Effect of temperature and related thermodynamic characteristics

Table 1. Influence of temperature on the enantioseparation of racemic of

\begin{tabular}{|c|c|c|c|}
\hline Temperature $\left({ }^{\circ} \mathrm{C}\right)$ & $D_{D}$ & $D_{L}$ & $\beta$ \\
\hline 15.0 & 10.42 & 4.2879 & 2.4303 \\
\hline 25.0 & 10.20 & 4.2028 & 2.4271 \\
\hline 35.0 & 10.15 & 4.1832 & 2.4264 \\
\hline 45.0 & 10.08 & 4.1554 & 2.4258 \\
\hline 55.0 & 10.03 & 4.1364 & 2.4248 \\
\hline
\end{tabular}

Concerntration of $D, L$-OFLX, 200ppm; D2EHPA, 0.3mol/L; L-(-)DTTA, $0.18 \mathrm{~mol} / \mathrm{L}$, L-(-)-DBTA, $0.12 \mathrm{~mol} / \mathrm{L}$; pHeq, 6.86 .

Temperature is on doubt an important factor in controlling chiral recognition processed. So the effect of temperature within a reasonable range was investigated, and calculated it accurately to one place of decimal. Table 1 shows that higher temperature leads to a decrease in both distribution ratios and enantioselectivities. Conventionally, according to Van't Hoff equation, the relationships of $D, \beta$ with thermodynamic parameters in the process of enantioselective separation are represented as

$$
\begin{aligned}
& \ln D=-\frac{\Delta H}{R T}+\frac{\Delta S}{R}+\ln \varphi \\
& \ln \beta=-\frac{\Delta \Delta H}{R T}+\frac{\Delta \Delta S}{R}
\end{aligned}
$$

where $R, T$ are the gas constant, the absolute temperature respectively. $\varphi$ is the phase ratio (volume ratio of the organic phase to aqueous phase). $\Delta H$ and $\Delta S$ are the enthalpy change and entropy change of the distribution of $D$-OFLX or $L$-OFLX between the organic phase and aqueous phase respectively, which can be obtained from linear regression of the Van't Hoff plots by plotting $\ln D$ versus $1 / T$ (Figure 7a, Figure7b). $\Delta \Delta H$ and $\Delta \Delta S$ are the enthalpy change and entropy change of the extraction resolution process of OFLX enantiomers, which can be calculated from the slope and intercept of linear portion of plot of $\ln \beta$ versus $1 / T$ (Figure $7 \mathrm{c}$ ). All the calculated results are listed in table 2 .

Table 2. The thermodynamic parameters for enantioselective extraction of racemic ofloxacin.

\begin{tabular}{|c|c|c|c|c|}
\hline Analyte & $\begin{array}{c}\Delta H(\mathrm{~J} \\
\left.\mathrm{mol}_{-1}\right)\end{array}$ & $\begin{array}{c}\Delta S(\mathrm{~J} \\
\left.\mathrm{mol}_{-1} \mathrm{~K}\right)\end{array}$ & $\Delta \Delta H\left(\mathrm{~J} \mathrm{~mol}_{-1}\right)$ & $\begin{array}{c}\Delta \Delta S(\mathrm{~J} \mathrm{~mol} \\
\mathrm{K})\end{array}$ \\
\hline$D$-OFLX & -441.06 & 17.83 & -26.58 & 7.28 \\
\hline$L$-OFLX & -414.70 & 10.54 & -28 \\
\hline
\end{tabular}
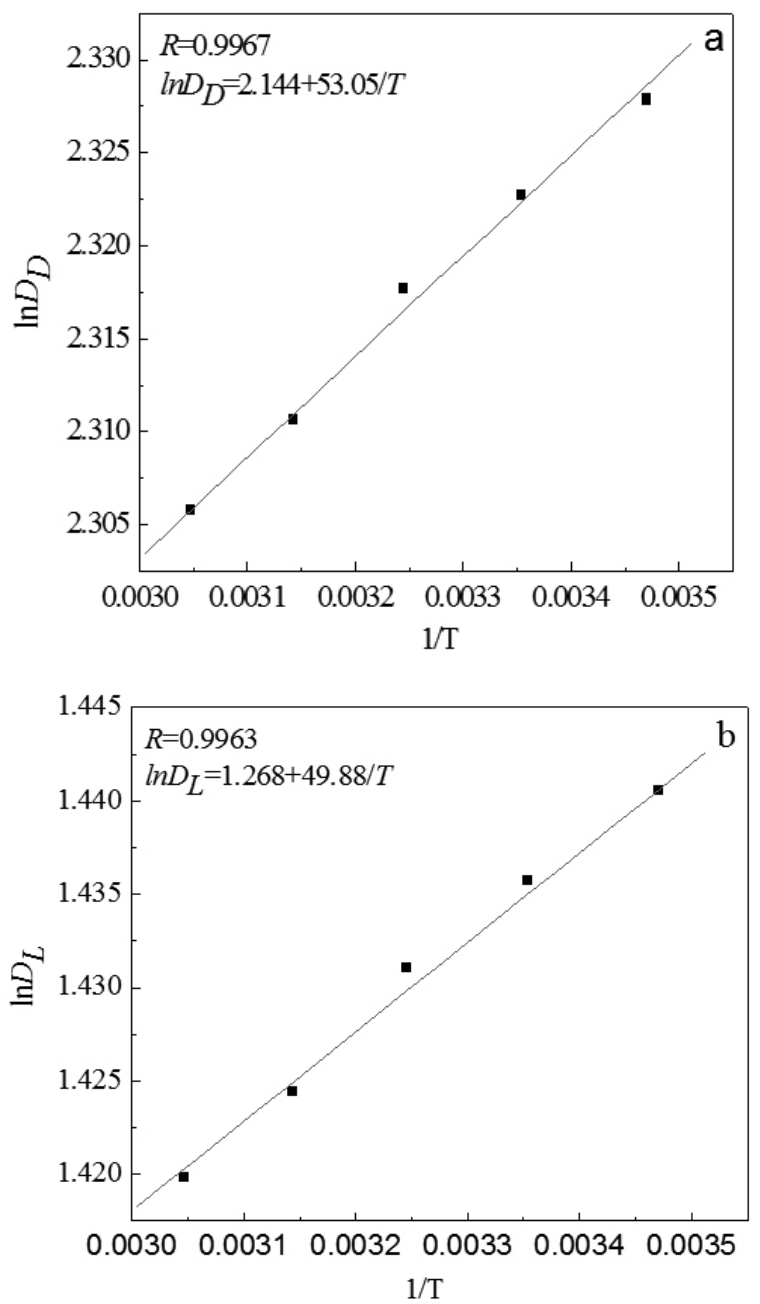


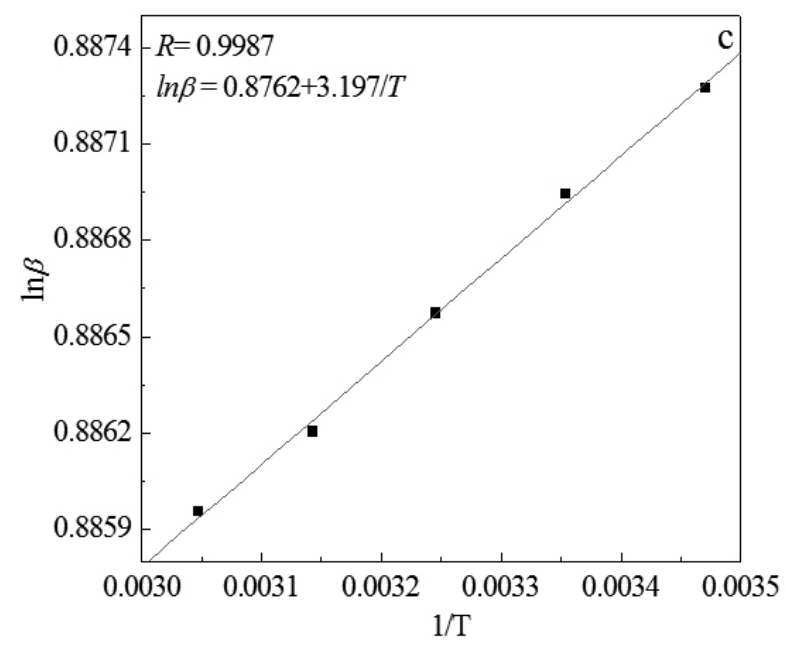

Figure 6: $\ln D_{D}(\mathrm{a}), \ln D_{L}(\mathrm{~b}), \ln \beta$ (c) vs. $1 / \mathrm{T}$ for the enantioseparation of ofloxacinn loxacin.

As shown in table $2, \Delta H<0$ means the process of extraction reaction is an exothermic process. $\Delta S>0$ indicates the extraction reaction is along with the increase of confusion degree of the whole extraction system. It is obvious that in the range of $288 \sim 330 \mathrm{~K}$, there always keeps $\Delta \Delta G<0$, which indicates a spontaneous process of extraction reaction. But in fact, the effects of extraction resolution was impaired due to the increase of confusion degree of the whole extraction system caused by increasing temperature. The plots in Figure 6 are well in conformity with the Van't Hoff model, from another point of view, indicating that the complexes do not change in conformation ${ }^{17,18}$ and that enantioselective interactions are unchanged in the temperature ranges studied ${ }^{19}$.

\section{CONCLUSION}

The mixed-extractants consisting of n-octanol and a mixed complex chiral selector formed from $L$-(-)-DBTA, $L$-(-)-DTTA and D2EHPA are found to be efficient in the extraction of oflxacin enantiomers, several factors including mixed complex chiral selector compositions, initial concentration of OFLX enantiomers, $\mathrm{pH}$ of aqueous phase and temperature, which influence the co-extraction efficiency are investigated. The results shows that the enantioselectivity has been enhanced compared with the individual complex chifal selectors. It is a useful way for searching new extractants or select appropriate pure isomer of racemic mixtures with both high extractive capacities and enantioselectivities, and it is also helpful to optimize the extraction systems and realize the large-scale production of the pure isomer of racemic mixtures with extraction method.

\section{ACKNOWLEDGEMENT}

We wish to acknowledge the support given to this work by the China National Natural Science Foundation (Project No. 20776162), and the Provincial Natural Science Foundation of Hunan (Project No. 09JJ3026).

\section{REFERENCES}

1.- R Herráez-Hernández, P Campíns-Falcó, J Chromatogr B. 740, 169 , (2000)

2.- S. Abolfazl Mostafavi, Robert T. Foster, Int J Pharm. 22, 97, (2000)

3.- B.Koppenhoefer, H. Allmendinger, Lueichang, J. Chromatogr. 89, 441, (1988)

4.- S Eto, H. Nada, A. Noda, J. Chromatogr. 253, 579, (1992)

5.- P. Hadik, L.-P. Szab' o, E. Nagy, Desalination. 148, 193, (2002)

6.- P. Paul, J. Chaudhuri, Chirality. 9, 261, (1997)

7.- Tan, B., Luo, G.S., Wang, J.D, Sep. Purif. Technol. 53(3), 330, (2007)

8.- Tan, B., Luo, G.S., Wang, J.D, Tetrahedron: Asymmetry. 17(6), 883, (2006)

9.- Pickering, P.J., Chaudhuri, J.B, Chirality. 9(3), 261, (1997)

10.- Zhao, S.L., Liu, Y.M, Electrophoresis. 22(13), 2769, (2001)

11.- Pietraszkiewicz, M., Koz biał, M., Pietraszkiewicz, O, J. Membr. Sci. 138(1), 109, (1998)

12.- De Bruin, T.J.M., Marcelis, A.T.M., Zuilhof, H., Rodenburg, L.M., Niederla "nder, H.A.G., Koudijs, A., Overdevest, P.E.M., Der Padt, A.V.; Sudho "1ter, E.J.R., Chirality. 12(8), 627, (2000)

13.- A. Péter, E. Vékes, G. Tóth, D. Tourwé, F. Borremans, J.Chromatogr. A. 948(1-2), 283, (2002)

14.- F. P. JIAO, X. Q. CHEN, YANG and Y. H. HU, Lat. Am. Appl. Res. 38 , $249,(2008)$

15.- F. P. JIAO, X. Q. CHEN and Z. WANG, Solvent Extraction and Ion Exchange. 27(4), 447, (2009)

16.- F. P. JIAO, X. Q. CHEN, X. Y. JIANG, Iran. J. Chem. Chem. Eng. 28, 1, (2009)

17.- Tan, B., Luo, G.S., Qi, X., Wang, J.D, Sep. Purif.Technol. 49(2), 186, (2006)

18.- M. Kazusaki, H. Kawabata, H. Matsukura, J. Liq. Chromatogr. Relat. Technol. 23, 2937, (2000)

19.- T.O'Brien, L.Crocker,R, Thompson, K. Thompson, P.H. Toma, D.A. Conlon, B. Feibush, C. Moeder, G. Bicker, N. Grinberg, Anal. Chem. 69, 1999, (1997) 